\title{
Osteitis Pubis: A Diagnosis for the Family Physician
}

\author{
Scott K. Andrews, MD, and Peter J. Carek, MD, MS
}

Background: Osteitis pubis was first described in 1924 in patients who had had suprapubic surgery. Since that time many theories concerning the cause of the disease have been developed. Published case reports and retrospective record reviews of specific, isolated patient populations have been used to postulate an infectious, inflammatory, or traumatic cause of this condition. Such confusion reduces the likelihood of an accurate diagnosis of osteitis pubis, particularly in the primary care setting, where it is becoming increasingly likely that patients afflicted with this frustrating illness will initially seek treatment.

Methods: This article describes a case report and provides a review of the literature. The medical literature was searched using the following key words: "abdominal pain," "pelvic pain," "inflammation," "symphysis pubis," and "enthesopathy."

Results and Conclusions: Osteitis pubis, considered to be the most common inflammatory disease of the pubic symphysis, is a self-limiting inflammation secondary to trauma, pelvic surgery, childbirth, or overuse, and it can be found in almost any patient population. Occurring more commonly in men during their $30 \mathrm{~s}$ and 40s, osteitis pubis causes pain in the pubic area, one or both groins, and in the lower rectus abdominis muscle. The pain can be exacerbated by exercise or specific movements, such as running, kicking, or pivoting on one leg, and is relieved with rest. Pain can occur with walking and can be in one or several of many distributions: perineal, testicular, suprapubic, inguinal, and postejaculatory in the scrotum and perineum. Symptoms are described as "groin burning," with discomfort while climbing stairs, coughing, or sneezing. A greater understanding and awareness of osteitis pubis will reduce patient and physician frustration while improving overall outcomes. (J Am Board Fam Pract 1998;11:291-5.)

Recurring pain is not an uncommon complaint of physically active people, both young and old. Though the cause of most activity-related problems is easily established, the origin of abdominal and pelvic pain can be relatively difficult to determine. The primary care physician must remember that the activity or sport in which the patient is participating and, more importantly, the actual motions undertaken by the individual patient engaged in that activity can be important factors when determining the leading possibilities and subsequent investigation. Osteitis pubis, an inflammatory condition involving the pubic symphysis, is a relatively common though infrequently diagnosed condition. Confusion with respect to its cause and treatment can lead to patient and physician frustration, and obtaining diagnostic tests can escalate expenses. Osteitis pubis should be consid-

Submitted, revised 17 September 1997.

From the Department of Family Medicine, Medical University of South Carolina, Charleston. Address reprint requests to Peter J. Carek, MD, MS, Department of Family Medicine, 171 Ashley Ave, Charleston, SC 29425. ered in active patients of any age with abdominal or pelvic pain in whom more obvious causes have been ruled out.

\section{Methods}

We describe a case report of a man with osteitis pubis. We then review the medical literature on abdominal pain and osteitis using the following key words: "abdominal pain," "pelvic pain," "inflammation," "symphysis pubis," "enthesopathy."

\section{Case Presentation}

A 44-year-old man came to the Family Medicine Center complaining of worsening midhypogastric and midline pelvic pain. The pain had developed gradually, and he reported no recent direct trauma or acute injury. Forced flexion at the waist and Valsalva maneuvers exacerbated his discomfort, but he did not experience pain at rest. The pain, described as a sharp, "stabbing" sensation, remained fairly localized to his upper pelvis and lower abdominal area.

The patient had no history of abdominal or 


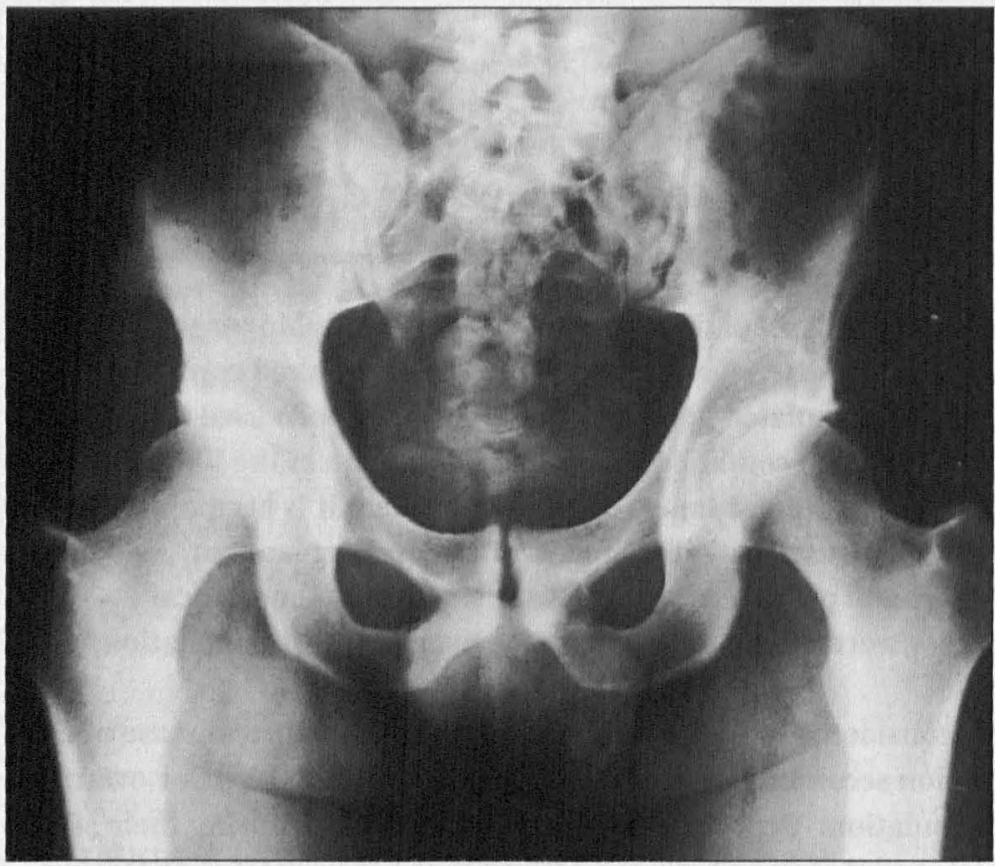

Figure 1. Plain radiographs of the pelvis revealing a slight widening and small amount of adjacent sclerosis of the symphysis pubis. though hip flexion, abduction, and external rotation (the FABER test) produced some pubic discomfort. Femoral pulses were 2+ bilaterally. There was no inguinal adenopathy or edema. Findings from neurologic and vascular examinations were within normal limits.

There were no clinically important findings on urinalysis, and no additional laboratory studies were ordered during the initial office visit.

Plain radiographs of the pelvis showed a slight widening and small amount of adjacent sclerosis of the symphysis pubis (Figure 1). Subsequent radionuclide imaging of the pelvic bone showed a small region of focally increased activity in the left pubic ramus consistent with an enthesopathy (inflammation at tendinous insertion into bone) (Figure 2).

The differential diagnosis of pubic symphysis tenderness includes muscle strain, prostatitis, orchitis, inguinal hernia, urolithiasis, ankylosing spondylitis, Reiter syndrome, hyperparathyroidism, metastasis, osteitis pubis, stress fracture, and rheumatoid arthritis. The findings for this patient's symphysis tenderness were consistent with osteitis pubis. Nonsteroidal anti-inflammatory medications were prescribed, and he was told to stop the offending activity (running) and given stretching exercises. He has been able to increase his exercise regimen gradually, though he has not yet returned to his previous level of physical activity. When offered local injection of an anesthetic and corticosteroid medications, he refused.

\section{Discussion}

In 1924 Beer, ${ }^{1}$ a urologist, first detailed osteitis pubis in patients after suprapubic surgery. Since that time osteitis pubis has been diagnosed frequently in patients after urologic or gynecologic surgery and in athletes, such as runners and soccer players. Many theories, from molecular to macroscopic, have been posited concerning the etiology and progression of the disease, but the cause of osteitis pubis remains unclear. Case reports and retrospective record reviews have been used to formulate hypotheses that the cause could be infectious, inflammatory, or traumatic. 
In support of the theory that it is due to trauma, osteitis pubis has been likened to gracilis syndrome, an avulsion fatigue fracture involving the bony origin of the gracilis muscle at the pubic symphysis and occurring in relation to the directional pull of the gracilis. ${ }^{2}$ Advances in radiographic studies have shown that osteitis pubis does not necessarily involve a fracture; therefore, it is an entity distinctly different from the gracilis syndrome. The process could be the result of stress reaction rather than direct trauma associated with fracture. This stress reaction might be associated with several biomechanical abnormalities (Table 1). ${ }^{3}$

Osteitis pubis has also been believed to have infectious origins. Infectious osteitis pubis occurs as a postoperative complication of urologic and gynecologic surgery in about 1 to 2 percent of cases. ${ }^{4}$ Pseudomonas aeruginosa is the most

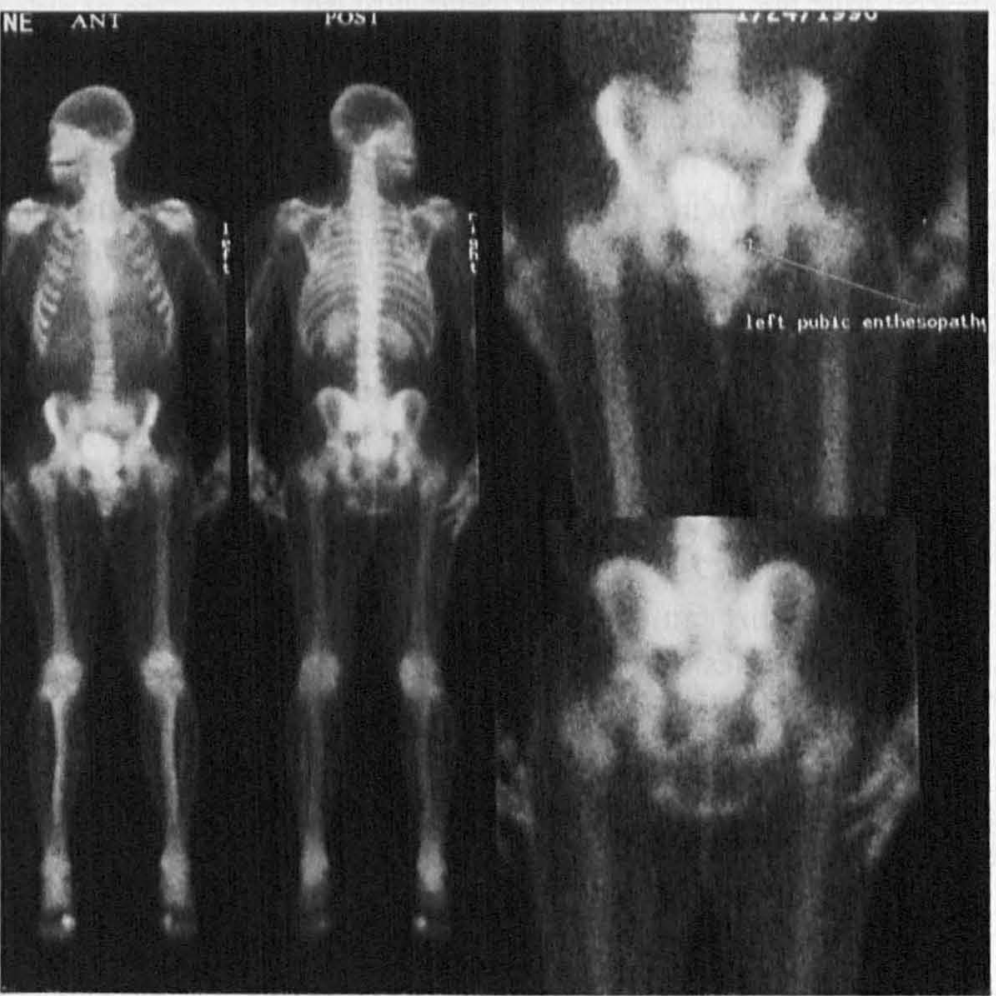

Figure 2. A bone scintiscan showing a small region of focally increased activity in the left pubic ramus consistent with an enthesopathy. common pathogen in infectious osteitis pubis and can be treated successfully with ciprofloxacin. Escherichia coli and Staphylococcus aureus have also been identified as causative organisms. Patients with postoperative osteitis pubis complain of fever and localized pain 1 month after surgery, and those patients with perioperative complications appear to have increased risk of developing infectious osteitis pubis.

Infectious osteitis pubis has also been diagnosed in intravenous drug abusers, with $P$ aeruginosa as the causative organism. In these patients the white cell count can be normal and the erythrocyte sedimentation rate only mildly elevated. Bacteremia is rarely found. Although there are no other signs of systemic illness, symptoms similar to those of osteitis pubis from other causes are common. Fineneedle aspiration might be inadequate, and bone biopsy has been required in some cases to establish the definitive diagnosis, the causative agent, ${ }^{5}$ and, therefore, the appropriate treatment choice.

Osteitis pubis has been studied in association with herniorrhaphy ${ }^{6}$ and urologic procedures, particularly suprapubic prostatectomy and cystectomy in men in their fifth to seventh decades, ${ }^{7}$ and has been found to have characteristics of an inflamma-

tory process. Biopsies of the affected area show signs of subacute and chronic inflammation involving the periosteum, bone, and cartilage. An inflammatory exudate of plasma cells and eosinophils also occurs.

Because the differential diagnoses and the etiologic spectrum are so broad, the case reports that have been published vary considerably. In one case of osteitis pubis a 16-year-old boy complained of acute abdominal pain, simulating acute appendicitis of a pelvic appendix. ${ }^{7}$ Osteitis pubis has been mistaken for chondrosarcoma in a patient with renal failure. ${ }^{8}$ Because pain from osteitis pubis can mimic prostatic pain, a patient actually had an or-

\section{Table 1. Proposed Biomechanical Causes of Osteitis Pubis.}

Excessive frontal or up-and-down pelvic motion or horizontal side-to-side sway

Instability of the sacroiliac joint leading to secondary stress reaction at the symphysis pubis

Limitation of hip movement with resultant shearing stress being applied to the hemipelvis on the opposite side

Repetitive adductor muscle pull at their origins on the inferior pubic rami 
chiectomy and another had a transurethral resection of the prostate before the true condition was diagnosed. ${ }^{9}$ Osteitis pubis has been misdiagnosed in patients who had postoperative pubic osteomyelitis following uterine suspension, suprapubic vaginal suspension, and a total abdominal hysterectomy-Buch procedure. ${ }^{10}$

Osteitis pubis is now considered to be the most common inflammatory disease of the pubic symphysis. It is a self-limiting inflammation secondary to trauma, pelvic surgery, childbirth, or overuse, and it can be found in almost any patient population.

Osteitis pubis can develop in athletes participating in activities that create continual shearing forces at the pubic symphysis, as with unilateral leg support, or acceleration-deceleration forces required during multidirectional activities. ${ }^{11}$ Soccer referees run 6 to 10 miles during any single game in a discontinuous, multidirectional manner. Whereas injury to soccer players is well documented, injuries incurred by soccer referees are not well described. ${ }^{12}$ Because they are involved in little contact, referees are presumably at risk for chronic overuse injuries. Occasionally osteitis can develop acutely subsequent to forced abduction at the hip, forced hip rotation, kicking, or trauma associated with a fall.

Osteitis pubis usually appears during the third and fourth decade of life and occurs more commonly in men. ${ }^{3}$ The pain or discomfort can be located in the pubic area, one or both groins, and in the lower rectus abdominis muscle. Symptoms of osteitis pubis have been described as "groin burning," with discomfort while climbing stairs, coughing, or sneezing. The pain can be exacerbated by exercise or specific movements, such as running, kicking, or pivoting on one leg, and relieved with rest. Pain with walking can be in one or several of many distributions: perineal, testicular, suprapubic, inguinal, and postejaculatory pain in the scrotum and perineum.

During the physical examination pain can be elicited by having the patient squeeze a fist between the knees with resisted long and flexed adductor contraction. Range of motion in one or both hips can be decreased. Adductor muscle spasm might occur with limited abduction and a positive lateral compression test and positive cross-leg test. ${ }^{13} \mathrm{~A}$ waddling antalgic gait, symphysis tenderness, and pain with adduction can also occur. A soft tissue mass with calcification and an audible or palpable click over the symphysis might be detected during daily activities. Plain radiographs can be normal early on, or there might be slight separation of the pubic bones with patchy sclerosis and irregular cortical margins. On radionuclide imaging of the bone, there is unilateral uptake in the pubic symphysis. ${ }^{14}$ Healing is characterized by gradual reossification with complete restoration occurring after many months. ${ }^{15}$

The differential diagnosis of nontraumatic groin pain in soccer participants, both players and referees, includes stress fractures, injury or diseases of the hip, osteitis pubis, nerve entrapment, and hernias. As in our patient, plain radiographs and a bone imaging will confirm the diagnosis.

Initial therapy should focus on decreasing inflammation with active rest, ice, nonsteroidal antiinflammatory drugs, and physical therapy. If necessary, judicious use of injected corticosteroids may be attempted. After pain and inflammation are alleviated, patients should start stretching the hip flexors and all the muscles about the hip and pelvis. A graduated program of exercises that place minimal torque across the pelvis, such as rowing or swimming, can then be slowly integrated. When discomfort has completely resolved, patients may progress to half-squats and leg presses with many repetitions to increase flexibility and endurance. After patients are asymptomatic during flexibility and strengthening exercises, they may be allowed to cycle and then attempt running. When patients are pain-free with restricted activities, they may return to full sports participation. A recurrence rate of 25 percent has been reported, with abandonment of the activity occurring in as high as 25 percent of those affected. The average return time to preinjury level of functioning is 3 to 6 months. ${ }^{16}$

\section{Conclusion}

Until recently osteitis pubis has been described only in association with certain surgical procedures, particularly urologic and gynecologic procedures. Recently osteitis pubis has been observed in professional and Olympic athletes. Our case report shows that osteitis pubis can occur outside these settings, where it might be encountered more frequently by primary care physicians. This disease can be diagnosed and treated by primary care physicians without the need for referral. Be- 
cause of its chronic nature, osteitis pubis is extremely frustrating for both patient and physician. Primary care physicians are in an ideal position to care for patients with this problem by providing continued encouragement and support, as well as education regarding prevention.

\section{References}

1. Beer E. Periostitis of the symphysis and descending rami of the pubes following suprapubic operations. Int J Med Surg 1924;37(5):224-5.

2. Wiley JJ. Traumatic osteitis pubis: the gracilis syndrome. Am J Sports Med 1983;11:360-63.

3. Fricker PA, Tauton JE, Ammann W. Osteitis pubis in athletes. Infection, inflammation, or injury? Sports Med 1991;12:266-79.

4. Desmond N, Bignardi GE, Coker RJ, Grech P, Harris JR. Infectious osteitis pubis in an HIV seropositive female. Genitourin Med 1994;70:127-9.

5. Magarian GJ, Reuler JB. Septic arthritis and osteomyelitis of the symphysis pubis (osteitis pubis) from intravenous drug use. West J Med 1985 142: 691-4.

6. Harth M, Bourne RB. Osteitis pubis: an unusual complication of herniorrhaphy. Can J Surg 1981;24: 407-9.

7. Pizzarello LD, Golden GT, Shaw A. Acute abdomi- nal pain caused by osteitis pubis. Am Surg 1974;40: 660-1.

8. Schabel SI, Burgener FA. Osteitis pubis in renal failure simulating chondrosarcoma. $\mathrm{Br} \mathrm{J}$ Radiol 1975; 48:1027-8.

9. Buck AC, Crean DM, Jenkins IL. Osteitis pubis as a mimic of prostatic pain. Br J Urol 1982;54:741-4.

10. Sexton DJ, Heskestad L, Lambeth WR, McCallum R, Levin LS, Corey GR. Postoperative pubic osteomyelitis misdiagnosed as osteitis pubis: report of four cases and review. Clin Infect Dis 1993;17:695700.

11. Sing R, Cordes R, Siberski D. Osteitis pubis in the active patient. Phys Sports Med 1995;23(12):67-73.

12. Garrett WE, Kirkendall DT, Contiguglia SR. The U.S. soccer sports medicine book. Baltimore: Williams \& Wilkins, 1996.

13. Grace JN, Sim FH, Shives TC, Coventry MB. Wedge resection of the symphysis pubis for the treatment of osteitis pubis. J Bone Joint Surg Am 1989;71:358-64.

14. Burke G, Joe C, Levine M, Sabio H. Tc-99 bone scan in unilateral osteitis pubis. Clin Nucl Med 1994;19:535.

15. McMurtry CT, Avioli LV. Osteitis pubis in an athlete. Calcif Tissue Int 1986;38:76- 7.

16. Batt ME, McShane JM, Dillingham MF. Osteitis pubis in collegiate football players. Med Sci Sports Exerc 1995;27:629-33. 